\title{
Dependência da prática de exercícios físicos: estudo com maratonistas brasileiros
}

\author{
Daniel Alves Rosa, Marco Túlio de Mello e Maria Lucia Oliveira Souza-Formigoni \\ Escola Paulista de Medicina - Departamento de Psicobiologia \\ São Paulo, SP - Brasil
}

\begin{abstract}
RESUMO
O presente estudo teve como objetivo testar, numa amostra de maratonistas brasileiros, a versão em português da adaptação da Escala de Dependência de Corrida proposta por Hailey e Bailey (1982). Métodos e resultados: 59 maratonistas de uma equipe da cidade de São Paulo foram abordados e orientados a preencher a Escala de Dependência de Corrida (EDC). A amostra foi composta, na sua maior parte, por homens (72\%) com média de $34 \pm 7$ anos, sendo que $77 \%$ corriam habitualmente havia cerca de dois a oito anos; $42,5 \%$ corriam de quatro a cinco vezes/semana e $81 \%$ dedicavam-se de uma a duas horas/dia em média para seus treinos. A média na pontuação total da EDC foi de $5 \pm 2,5$ pontos (escala 0-14 pontos). A correlação entre a pontuação total da EDC com cada uma das 23 questões que compõem o instrumento revelou que 10 questões apresentaram níveis de correlação significativos. As respostas positivas que apresentaram maior sensibilidade foram: "Sinto que me falta algo quando não corro" $(\mathrm{r}=0,61)$; "A corrida tem influenciado meu estilo de vida" $(r=0,58)$ e "Experimento grande prazer quando corro" $(\mathrm{r}=0,56)$. Conclusão: $\mathrm{Ob}-$ servamos na amostra brasileira níveis médios de pontuação na escala semelhantes aos descritos pelos autores do instrumento original, sugerindo que a tradução não alterou a sensibilidade da escala e que este instrumento possa ser útil no estudo da dependência da prática de corrida (ou exercícios físicos) em desportistas brasileiros.
\end{abstract}

Palavras-chave: Dependência. Maratonista. Questionário. Exercício físico.

Recebido em 17/10/02

Aceito em 28/12/02

Endereço para correspondência:

Daniel Alves Rosa

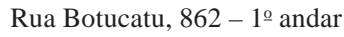

04023-062 - São Paulo, SP - Brasil

E-mail: daniel@psicobio.epm.br

\section{ABSTRACT}

Exercise dependence: a study with Brazilian marathon runners

This study aimed at testing, in a sample of Brazilian marathon runners, the Brazilian adaptation of the Negative Addiction Scale (Haley \& Bailey, 1982). Methods: 59 marathon runners of a team from São Paulo were asked to fill out the Brazilian version of the Negative Addiction Scale. Most of the sample was made up by men (72\%) aged $34 \pm$ 7; 77\% of whom had been running four to five times a week (42.5\%) for two to eight years; $81 \%$ spent one to two hours a day training. The average score in the Negative Addiction Scale was $5.2 \pm 2.5$ (the scale scores from 0 to 14). The correlation between total score in the scale and each of the 23 questions was significant in 10 of them. The positive answers which presented higher sensitivity were: "I feel something is missing when I don't run" ( $r=0.61)$; "Running has influenced my lifestyle" $(r=0.58)$, and "I experience a great pleasure when I run" $(r=0.56)$. Conclusion: The mean score in the Brazilian sample was similar to that described by the authors of the original instrument, suggesting that the translation did not affect the sensitivity of the instrument and that it can be useful in the study of running or physical activity dependence in Brazilian athletes.

Key words: Dependence. Marathon runners. Negative addiction scale. Physical exercise.

\section{INTRODUÇÃO}

Embora a prática regular de exercícios físicos resulte em benefícios físicos e psicológicos ${ }^{1,2}$, nos últimos anos deuse grande atenção ao fato de que a prática excessiva de exercícios poderia ser ou desencadear um comportamento compulsivo. A compulsão por exercícios físicos tem recebido grande atenção dos pesquisadores, desde o primeiro relato referente a esse fenômeno, descrito por Baekeland, em $1970^{3}$. Ao estudar o padrão de sono em praticantes de 
exercícios após um período de abstinência, Baekeland ${ }^{3}$ constatou que alguns indivíduos se recusavam a suspender seus programas de exercícios, mesmo quando lhes era oferecido dinheiro para participação no estudo. Após esse relato, inúmeros termos foram aplicados para descrever tal fenômeno. Glasser ${ }^{4}$ usou o termo positive addiction (dependência positiva) baseado na idéia de que a prática regular de exercícios físicos leva a alterações físicas e psicológicas benignas. Entretanto, Morgan ${ }^{5}$ utilizou o termo negative addiction, ao considerar que a prática excessiva de exercícios está associada a aspectos prejudiciais à saúde física e mental do indivíduo. Lyons e Cromey ${ }^{6}$ correlacionaram o excesso da prática de exercícios a sintomas de transtornos alimentares, usando o termo compulsive jogging para descrever sua pesquisa. DeCoverley Veale ${ }^{7}$ propôs o termo "dependência de exercício", por considerá-la similar aos demais tipos de dependência.

Segundo DeCoverley Veale ${ }^{7}$, a dependência de exercício poderia ser caracterizada pelos seguintes critérios: “1) estreitamento do repertório, levando a um padrão estereotipado de exercícios uma ou mais vezes por dia; 2) saliência do comportamento de praticar exercícios, dando prioridade sobre outras atividades, para que seja mantido o padrão de exercícios; 3) aumento na tolerância à quantidade e freqüência dos exercícios com o decorrer dos anos; 4) sintomas de abstinência relacionados a transtornos de humor (irritabilidade, depressão, ansiedade, etc.), quando interrompida a prática de exercícios; 5) alívio ou prevenção do aparecimento de síndrome de abstinência por meio da prática de mais exercícios; 6) consciência subjetiva da compulsão pela prática de exercícios; 7) rápida reinstalação dos padrões prévios de exercícios e sintomas de abstinência após um período sem prática de exercícios".

Outras características associadas à dependência de exercício são: "1) o indivíduo continua a prática de exercícios, mesmo quando se apresenta doente, lesionado ou com qualquer outra contra-indicação médica, ou a prática de exercícios físicos interfere negativamente nos relacionamentos com o companheiro, familiares, amigos ou no trabalho; 2) o indivíduo faz dieta alimentar para perda de peso como um meio de melhorar o desempenho"7.

De acordo com Morris et al. ${ }^{8}$, alguns corredores apresentavam sintomas de abstinência, tais como irritabilidade, ansiedade, depressão e sentimentos de culpa, quando eram impedidos de participar de suas rotinas de corridas regulares. Entre as evidências que fortalecem a hipótese da existência de dependência de exercício, encontram-se relatos de corredores sobre a interferência da prática regular de corrida no convívio familiar, social e no ambiente de trabalho ${ }^{9}$. Rolf ${ }^{10}$ relata ser comum a existência de vários tipos de lesões músculo-esqueléticas e/ou demais compli- cações devidas a cargas excessivas de exercícios físicos, em indivíduos que praticam exercícios físicos com alta freqüência e/ou intensidade.

Embora a síndrome de dependência de exercício possa gerar complicações à saúde física e mental do indivíduo, poucos são os instrumentos desenvolvidos para o estudo deste fenômeno. Destaca-se o Negative Addiction Scale ${ }^{11}$, que visa quantificar o grau de dependência, com base nos aspectos negativos da prática de corrida, focalizando principalmente as características psicológicas da dependência.

Visto que existem poucos estudos nacionais sobre esse fenômeno, o presente teve como objetivo testar, numa amostra de maratonistas brasileiros, a sensibilidade da versão traduzida da escala de dependência negativa de corrida elaborada por Hailey e Bailey ${ }^{11}$ e comparar os dados em uma amostra brasileira com os apresentados no estudo original.

\section{METODOLOGIA}

\section{Escala de dependência de corrida}

\section{Etapa de tradução}

Após autorização das autoras da versão original do $\mathrm{Ne}$ gative Addiction Scale, foi realizada sua tradução para o português falado no Brasil, por dois pesquisadores familiarizados com o conteúdo envolvido no material da linguagem fonte. Para a confiabilidade da tradução da Negative Addiction Scale, foi feita uma retrotradução (backtranslation), para garantir a obtenção de equivalência semânti$\mathrm{ca}^{12,13}$. A retrotradução, realizada por um tradutor bilíngüe, que não possuía conhecimento prévio do material, foi examinada por dois pesquisadores, que compararam cuidadosamente os itens nas duas versões (a original e a retrotraduzida) e na tradução para o português, para determinar se o significado preciso do inglês se havia mantido. Isso, segundo Brislin ${ }^{14}$, é uma condição indispensável para um processo adequado de tradução.

A retrotradução e adaptação da Negative Addiction Scale (quadro 1) foi enviada às autoras do instrumento original, que aprovaram e autorizaram sua utilização no presente estudo. O instrumento avalia os aspectos psicológicos "negativos" da dependência de corrida, por meio de uma escala de 14 itens, atribuindo-se a cada item um escore $(0$ ou 1). Escores altos estão associados a maiores níveis da dependência de corrida. Além da escala, foram acrescentadas questões sobre dados sociodemográficos e outras variáveis relacionadas à rotina de treinamento.

\section{CASUÍSTICA}

A pesquisa foi realizada em uma amostra de critério e conveniência composta por 59 maratonistas de ambos os sexos. Após assinarem um consentimento de participação 


\section{QUADRO 1}

\section{Questionário de Dependência da Prática de Corrida}

1. Durante uma semana normal eu corro:
a) Todos os dias
b) 6 dias
c) 5 dias
d) 4 dias
e) É variável

2. Nos dias em que não corro, normalmente me sinto:
a) tenso
b) culpado
c) igual aos outros dias nos quais corro
d) outros (especificar)

3. Desde que comecei a praticar corrida meu interesse em divertir-me em outras atividades sociais tem:
a) aumentado
b) diminuído
c) manteve-se o mesmo

4. No dia em que não corro, sinto-me deprimido ou mentalmente devagar.
(1) concordo muito
(2) concordo moderadamente
(3) concordo pouco
(4) discordo pouco
(5) discordo muito

5. No dia em que não corro, sinto que me falta algo.
(1) concordo muito
(2) concordo moderadamente
(3) concordo pouco
(4) discordo pouco
(5) discordo muito

6. Se parasse de correr minha saúde física declinaria significativamente.
(1) concordo muito
(2) concordo moderadamente
(3) concordo pouco
(4) discordo pouco
(5) discordo muito

7. Correr é minha principal forma de recreação.
(1) concordo muito
(2) concordo moderadamente
(3) concordo pouco
(4) discordo pouco
(5) discordo muito

8. Experimento um alto nível de prazer na maioria das minhas sessões de corrida.

(1) concordo muito

(2) concordo moderadamente

(3) concordo pouco

(4) discordo pouco

(5) discordo muito
9. A corrida é um assunto comum em minhas conversas.

(1) concordo muito

(2) concordo moderadamente

(3) concordo pouco

(4) discordo pouco

(5) discordo muito

10. É importante para todos aqueles que correm, interromperem por algum tempo suas rotinas de corrida.

(1) concordo muito

(2) concordo moderadamente

(3) concordo pouco

(4) discordo pouco

(5) discordo muito

11. A corrida tem influenciado meu estilo de vida.
(1) concordo muito
(2) concordo moderadamente
(3) concordo pouco
(4) discordo pouco
(5) discordo muito

12. Meu interesse pela corrida tem causado alguns problemas nas minhas relações familiares e interpessoais.
(1) concordo muito
(2) concordo moderadamente
(3) concordo pouco
(4) discordo pouco
(5) discordo muito

13. Marque apenas as afirmativas que se aplicam ao seu comportamento de correr.

A) Eu corro aproximadamente no mesmo horário todos os dias.

B) Eu corro em ambientes desfavoráveis (ex.: chuva, frio, calor)

C) Tenho um programa semanal de corrida constante, que possui o mesmo padrão com dias nos quais corro e dias nos quais não corro.

D) Eu corro considerando se aquele horário do dia é o mais conveniente, em consideração às minhas outras atividades diárias.

E) Tenho um companheiro de treinamento com o qual eu pratico minhas sessões de corrida sempre que possível.

F) Eu mantenho um registro por escrito de minhas sessões de corrida.

G) Eu planejo minhas outras atividades baseado no tempo que quero correr.

H) Sou normalmente disciplinado e corro mesmo nos dias que realmente não estou disposto a correr.

I) Eu estabeleço metas para minhas sessões de corrida da semana.

J ) Eu sou capaz de alcançar as metas das minhas sessões de corrida que planejei para a semana.

K) Sinto que, se não mantiver minha autodisciplina, pararia completamente de correr no dia seguinte.

Pontuação: é atribuído um (1) ponto para toda resposta "concordo muito" nas questões 4 até 9 e nas questões 11 e 12 . Na questão 10 é atribuído um (1) ponto para resposta "discordo muito". Atribui-se um (1) ponto se as questões 1 =a; 2 =a ou b e 3 =b. Para cada resposta afirmativa nos itens B e/ou H na questão 13 deve-se atribuir um ponto. A soma dos pontos de todo o questionário representa a pontuação total obtida. 
do qual constavam os objetivos da pesquisa, era solicitado aos atletas que preenchessem o questionário e o entregassem ao coordenador do estudo, sendo garantido total sigilo de suas respostas. O projeto foi aprovado pelo Comitê de Ética em Pesquisa da Unifesp.

\section{Análise estatística}

A correlação entre a pontuação total e cada uma das respostas da Escala de Dependência de Corrida foi analisada pelo coeficiente de correlação de Spearman. Para a comparação dos resultados encontrados no presente estudo com os do estudo original, utilizamos o teste $t$ de Student. O nível de significância estatística estabelecido para todos os testes foi de 5\%, tendo sido utilizado o software Statistica (Statsoft, Inc., 1999).

\section{RESULTADOS}

Os dados sociodemográficos da amostra são apresentados na tabela 1. A maioria da amostra $(51 \%)$ apresentava nível superior completo de escolaridade, independentemente do sexo analisado e trabalhava e/ou estudava.

A tabela 2 apresenta as características da amostra de acordo com a freqüência semanal e tempo (horas diárias dedicadas à prática de corrida e anos de prática). Observa-se que a maioria dos atletas $(52 \%)$ relatou no mínimo dois anos ininterruptos de prática de corrida. Nota-se também que $28,6 \%$ dos maratonistas do sexo masculino relataram de quatro a oito anos de prática de corrida. Cerca de $81 \%$ dos entrevistados dedicavam-se de uma a duas horas/dia às suas rotinas de treinos para a corrida. Quanto à frequiência semanal, 32,2\% dos atletas relataram correr menos de quatro vezes por semana e, a grande maioria, em frequiência igual ou superior a quatro vezes por semana.

A pontuação média na EDC apresentada pelo total da amostra foi de $5 \pm 2,5$ (média \pm desvio padrão), não havendo diferenças estatisticamente significativas na comparação entre os sexos, visto que os homens apresentaram pontuação média de 5,0 $\pm 2,6$ pontos, enquanto que nas mulheres a média foi de $5,4 \pm 2,0$ pontos (figura 1).

Na tabela 3 são apresentadas as médias obtidas na EDC dos homens da amostra brasileira que relataram mais de quatro anos de corrida e as da amostra relatada por Hailey e Bailey (1982). Não houve diferenças estatisticamente significativas entre as médias obtidas na EDC pelos homens

2 a 3

$>12$
TABELA 1

Distribuição da amostra de acordo com as características demográficas

\begin{tabular}{lccc}
\hline & \multicolumn{2}{c}{ Sexo } & \\
\cline { 2 - 3 } & $\begin{array}{c}\text { Masculino } \\
\mathbf{n = 4 2}\end{array}$ & $\begin{array}{c}\text { Feminino } \\
\mathbf{n = 1 7}\end{array}$ & $\begin{array}{c}\text { Total } \\
\mathbf{n = 5 9}\end{array}$ \\
\hline Idade (anos) & $35 \pm 7$ & $29 \pm 4$ & $34 \pm 7$ \\
\hline Escolaridade máxima (\%) & & & \\
$\quad$ 10 grau incompleto ou completo & 7,0 & 6,0 & 7,0 \\
2o grau incompleto ou completo & 26,5 & 12,0 & 22,0 \\
Superior incompleto ou completo & 66,5 & 82,0 & 71,0 \\
\hline $\begin{array}{l}\text { Ocupação (\%) } \\
\text { Trabalha }\end{array}$ & 98 & & \\
Estuda & 33 & 35 & 34 \\
\hline
\end{tabular}

TABELA 2

Características da prática de comida da amostra brasileira (\%)

Prática de corrida

\begin{tabular}{|c|c|}
\hline \multicolumn{2}{|c|}{ Sexo } \\
\hline $\begin{array}{c}\text { Masculino } \\
n=42\end{array}$ & $\begin{array}{c}\text { Feminino } \\
n=17\end{array}$ \\
\hline
\end{tabular}

Tempo (anos)

$\begin{array}{rrr}47,6 & 76,4 & 55,9 \\ 38,1 & 17,6 & 32,2 \\ 14,3 & 6,0 & 11,9\end{array}$

Duração das sessões (horas /dia)

$\begin{array}{llll}3 \text { a } 4 & 83,3 & 76,5 & 81,3 \\ & 16,7 & 23,5 & 18,7\end{array}$

\begin{tabular}{lrrr}
\hline Freqüência semanal (dias/semana) & & & \\
$<4$ & 33,3 & 29,4 & 32,2 \\
4 & 16,7 & 29,4 & 20,3 \\
5 a 6 & 45,2 & 41,2 & 44,1 \\
7 & 4,8 & 0,0 & 3,4 \\
\hline
\end{tabular}

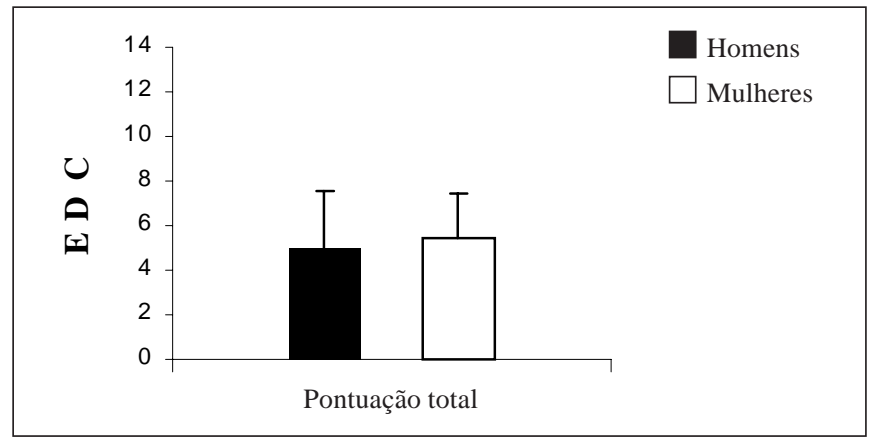

Fig. 1 - Pontuação da amostra brasileira na Escala de Dependência de Corrida (EDC - média \pm desvio padrão) dos corredores classificados de acordo com o sexo 
TABELA 3

Níveis médios de pontuação na Escala de Dependência de Corrida (EDC) relatados no estudo de Hailey e Bailey (1982) e na amostra brasileira, classificados de acordo com o tempo de prática

\begin{tabular}{|c|c|c|c|c|c|}
\hline \multirow{3}{*}{$\begin{array}{l}\text { Tempo de } \\
\text { prática }\end{array}$} & \multicolumn{4}{|c|}{ Amostra } & \multirow{3}{*}{$\begin{array}{c}\text { P (amostra americana } \\
\text { vs. brasileira) }\end{array}$} \\
\hline & \multicolumn{2}{|c|}{ Americana } & \multicolumn{2}{|c|}{ Brasileira } & \\
\hline & Indivíduos & EDC & Indivíduos & EDC & \\
\hline$<4$ anos & 32 & $5,6 \pm 2,4$ & $\mathrm{n}=20$ & $4,8 \pm 2,2$ & 0,24 (n.s) \\
\hline$>4$ anos & 16 & $7,0 \pm 2,6$ & $\mathrm{n}=22$ & $5,3 \pm 3,1$ & 0,07 (n.s) \\
\hline
\end{tabular}

positivas às seguintes afirmativas: "Sinto que me falta algo quando não corro" $(\mathrm{r}=0,61)$; "A corrida tem influenciado meu estilo de vida" $(r=0,58)$ e "Experimento grande prazer quando corro" ( $\mathrm{r}=$ $0,56)$ (tabela 4).

\section{DISCUSSÃO}

Os resultados apresentados neste estudo demonstram que os valores médios de pontuação na

TABELA 4

Níveis de correlação ( $r$ de Spearman) entre a pontuação total na EDC e cada questão. As questões não apresentadas não atingiram níveis de correlação estatisticamente significativos

\section{Questões}

(rs)

1. Durante uma semana normal eu corro todos os dias.

2. Nos dias em que não corro, normalmente me sinto tenso ou culpado.

3. Desde que comecei a praticar corrida meu interesse em divertir-me em outras atividades sociais tem diminuído.

4. No dia em que não corro, sinto-me deprimido ou mentalmente devagar.

5. No dia em que não corro, sinto que me falta algo.

7. Correr é minha principal forma de recreação.

8. Eu experimento um alto nível de prazer na maioria das minhas sessões de corrida.

9. A corrida é um assunto comum em minhas conversas.

11. A corrida tem influenciado meu estilo de vida.

13. Eu sou normalmente disciplinado e corro mesmo nos dias em que realmente não estou disposto a correr.

do estudo original e pelos da presente amostra $(\mathrm{p}<0,05)$. Tanto no estudo original quanto na amostra brasileira observou-se que os valores médios da EDC eram relativamente maiores no grupo que relatava dedicar-se mais de quatro anos à prática de corrida.

A correlação entre a pontuação total da EDC e cada uma das 23 questões revelou que 10 apresentaram níveis significativos de correlação, sendo mais sensíveis as respostas escala de dependência de corrida da amostra brasileira foram semelhantes aos apresentados no estudo de Hailey e Bailey $^{11}$. Aquelas autoras detectaram uma relação linear entre a média de pontos obtidos na escala de dependência e a quantidade de anos de prática regular de corrida. Elas observaram que corredores com menos de um ano de prática de corrida apresentavam menor pontuação total na escala de dependência de corrida do que aqueles com tempo de prática superior ${ }^{11}$.

Em nosso estudo, grande parte da amostra relatou praticar corrida por período não superior a dois anos, impossibilitando o estudo da relação entre tempo de envolvimento com a prática de corrida e intensidade de dependência. No entanto, observamos valores médios de dependência de corrida discretamente maiores nos indivíduos que relataram estar envolvidos por período superior a quatro anos com a prática de corrida. Esses dados estão de acordo com outros estudos, como de Pierce et al. ${ }^{15}$, que demonstraram que atletas participantes de corridas de longas distâncias, que exigiam vários anos de dedicação, tais como maratona e ultramaratona, apresentavam maiores escores para dependência de corrida do que os participantes de provas com menores distâncias percorridas.

Diferentemente do primeiro estudo ${ }^{11}$, estudamos também uma amostra feminina ( $\mathrm{n}=17)$, que não diferiu da masculina quanto à pontuação total na escala de dependência de corrida. Esse resultado sugere que talvez não existam diferenças entre corredores e corredoras em relação ao desenvolvimento da dependência da prática de corrida. Nossos dados corroboram os de Furst e Germone ${ }^{16}$, que também relataram não haver diferenças na dependência de exercício entre homens e mulheres, para diversos tipos de atividades físicas. Entretanto, Crossman et al. ${ }^{17}$ relataram que homens apresentam maiores desconfortos (síndrome de abstinência) quando interrompem seus programas de treinamento do que mulheres. Outros trabalhos têm demons- 
trado que mulheres apresentam maiores escores de dependência de exercício do que homens, quando pareados em relação ao volume (quantidade) de treinamento ${ }^{18}$.

Segundo Masters e Lambert ${ }^{19}$, mulheres envolvidas em grandes rotinas de atividades físicas possuem menor aprovação social do que homens. Sendo assim, aquelas que persistem desenvolveriam maior percepção da dependência, devido às pressões sociais. Também tem sido sugerido que a maior incidência de transtornos alimentares entre mulheres explicaria os altos níveis de dependência de exercício, que podem ser associados a tais transtornos ${ }^{6}$.

Em relação à sensibilidade do questionário, pudemos observar que as questões mais sensíveis foram aquelas que se referem a percepção subjetiva do "compromisso" com a prática de corrida e, principalmente, com um possível sintoma de abstinência da prática de corrida. Thompson e Blanton ${ }^{20}$ propõem uma adaptação do modelo de "processo oponente" (opponent-process model) criado por Solomon ${ }^{21}$ para o desenvolvimento da dependência de exercício, com as seguintes fases: a) percepção afetiva ou hedônica das sessões de exercícios; b) desenvolvimento de tolerância aos estímulos provocados pelo exercício, desencadeando a necessidade de aumentar sua intensidade ou duração; e c) presença de síndrome de abstinência após a interrupção das sessões de exercícios.

Numerosos estudos têm demonstrado a presença de síndrome de abstinência em decorrência da interrupção das rotinas de exercícios entre praticantes regulares. Hauck e Blumenthal $^{22}$ demonstraram que $86 \%$ de uma amostra de corredores sentiam-se culpados se perdessem uma sessão de treinamento e $72 \%$ sentiam-se tensos, irritados ou deprimidos, se estivessem incapazes de correr por algum motivo. Sachs e Pargman ${ }^{9}$, usando entrevistas para o estudo da dependência de exercício em corredores, concluíram que a síndrome de abstinência parece ser um importante fator nesse fenômeno.

A maior sensibilidade da questão "Sinto que me falta algo quando não corro" encontrada em nosso estudo corrobora estudos que consideram os sintomas de abstinência uma das principais características da dependência de exercício.

Uma limitação deste estudo é o reduzido tamanho da amostra, fazendo-se necessária sua ampliação para estudar mais profundamente a questão. Entretanto, esses dados são sugestivos da existência da dependência de exercício.

\section{AGRADECIMENTOS}

Ao grupo Pão de Açúcar Club da cidade de São Paulo por permitir a realização deste trabalho com seus atletas. À Coordenação de Aperfeiçoamento de Pessoal de Nível Superior-Capes e ao Conselho Nacional de Pesquisa pelas bolsas concedidas aos pesquisadores e à Associação Fundo de Incentivo à Psicofarmacologia-AFIP, pelo apoio financeiro para realização deste trabalho.

Todos os autores declararam não haver qualquer potencial conflito de interesses referente a este artigo.

\section{REFERÊNCIAS}

1. Hughes JR. Psychological effects of habitual aerobic exercise: a critical review. Prev Med 1984;13:66-78.

2. Pate RR, Pratt M, Blair SN, Haskell WL, Macera CA, Bouchard C, et al. Physical activity and public health: a recommendation from the Centers for Disease Control and Prevention and the American College of Sports Medicine. JAMA 1995;273:402-7.

3. Baekeland F. Exercise deprivation. Sleep and psychological reactions. Arch Gen Psychiatry 1970;22:365-9.

4. Glasser W. Exercise addiction. New York: Harper \& Row, 1976.

5. Morgan WP. Negative addiction in runners. Phys Sports Med 1979;7: 56-63.

6. Lyons HA, Cromey R. Case report. Compulsive jogging: exercise dependence and associated disorder of eating. Ulster Med J 1989;58:100-2.

7. Decoverley Veale DMW. Exercise dependence. Br J Addict 1987;82: 735-40.

8. Morris M, Steinberg H, Sykes EA, Salmon P. Effects of temporary withdrawal from regular running. J Psychosom Res 1990;34:493-500.

9. Sachs M, Pargman D. Running addiction: a depth interview examination. Journal of Sport Behaviour 1979;2:143-55.

10. Rolf C. Overuse injuries of the lower extremity in runners. Scand J Med Sci Sports 1995;5:181-90.

11. Hailey BJ, Bailey LA. Negative addiction in runners: a quantitative approach. Journal of Sport Behaviour 1982;5:150-4.

12. Flaherty JA. Appropriate and inappropriate research methodologies for Hispanics mental health. Health \& behaviour: research agenda for hispanics. The Research Monography Series $N^{\circ}$. 1. The University of Illinois at Chicago, 1987.

13. Flaherty JA, Gaviria FM, Pathak D, Mitchell T, Wintrob R, Richiman JA, et al. Developing instruments for cross-cultural psychiatric research. J Nerv Ment Dis 1988;176:257-63.

14. Brislin RW. Back-translation for cross-cultural research. J Cross-Cultural Psychol 1970;1:185-216.

15. Pierce EF, McGowan RW, Lynn TD. Exercise dependence in relation to competitive orientation of runners. J Sports Med Phys Fitness 1993;33: 189-93.

16. Furst DM, Germone K. Negative addiction in male and female runners and exercisers. Percept Mot Skills 1993;77:192-4.

17. Crossman J, Jamieson J, Henderson L. Responses of competitive athletes to layoffs in training: exercise addiction or psychological relief? Journal of Sport Behaviour 1987;10:28-38.

18. Pierce EF, Rohaly KA, Fritchley B. Sex differences on exercise dependence for men and women in a marathon road race. Percept Mot Skills 1997;84:991-4.

19. Masters KS, Lambert MJ. On gender comparison and construct validity: an examination of the commitment to running scale in a sample of marathon runners. Journal of Sport Behaviour 1989;12:196-203.

20. Thompson JK, Blanton P. Energy conservation and exercise dependence: a sympathetic arousal hypothesis. Med Sci Sports Exerc 1987;19:91-9.

21. Solomon RL. The opponent-process theory of acquired motivation: the costs of pleasure and the benefits of pain. Am Psychol 1980;35:691712 .

22. Hauck ER, Blumenthal JA. Obsessive and compulsive traits in athletes. Sports Med 1992;14:215-27. 